\title{
Design and Implementation of Control Software Based on Linux and QT for Electric Energy Meter Calibration Device
}

\author{
Lei LUO *, Zhi-Wei HE and Jie WANG \\ College of Electron Information, Hangzhou Dianzi University, Hangzhou, China \\ luolei_hdu@163.com
}

\begin{abstract}
Keywords: Linux, Thread, Meter Calibration, Standard Electric Energy Meter, Error Checking Instrument, Signal Source.

Abstract. With the development of society, all the industrial fields are now dependent on electric energy. An energy electric meter is a special instrument for measuring electric energy, its accuracy and reliability have a huge impact on people's lives. To ensure the reliability and accuracy of electric energy meter measurement, it must be checked before or after the factory. In this paper, the design and implementation of control software based on Linux and QT for electric energy meter calibration are proposed. This greatly improves the accuracy and reliability of the system, reduces the cost of the whole system, and shortens the time required for the verification of the electric energy and also improves the efficiency of the verification. At the same time, the designed system also can meet the requirements of the original technical indicators. The accuracy of the device verification is also up to the grade of 0.05 .
\end{abstract}

\section{Introduction}

In the early days, we mainly adopted the manual verification method, it is difficult to meet the requirements of verification because of many practical reasons such as too many verification procedures and low efficiency. With the increase of user requirements, manual verification is no longer in line with actual requirements. Nowadays, the automatic verification is widely used to calibrate the electric energy meter. Due to the verification device requires the human-computer interaction interface, the application program based on single chip microcomputer can not satisfy the demand. So we think an embedded operating system should be chosen, apart from the general functions of the operating system, it should have the advantages of compactness, real-time and high stability. At present, the common operating systems are WinCE, VxWorks, Linux. In this, we have chosen the Linux system. Linux is a multi user, multi thread, and multi CPU operating system, its main features are:

(1) Linux is a free operating system. It has the rich software resources, its source code is completely shared. Users can compile their own kernel, modify and expand the operating system for the second-hand developments.

(2) The design of the Linux's kernel is exquisite and high degree of modularity. It is divided into memory management, interprocess communication, process scheduling, network interface and virtual file system. According to the needs of users, Linux can real-time insert some modules or remove them from the kernel, which is the reason why the Linux's kernel is ingenious and suitable for embedded system development.

(3) Linux has a wide range of hardware support. Its kernel is mostly written in C language, and it use the portable Unix standard application interface, and can run on multiple hardware platforms such as ARM, x86, amd64.

(4) Linux's security and reliability are good. It has high performance, its high performance show low occupancy in Linux system resources and advantages in high-performance computing. In addition, there are many enthusiastic groups and individuals involved in the development of Linux, you can get the latest security messages, and timely repair vulnerabilities in the system.

In addition, QT is a cross platform $\mathrm{C}++$ graphical user interface library. It is easy to learn, and has excellent cross platform, object-oriented, rich API interface, and a very good support for 2D/3D graphics rendering. The electric energy meter belongs to the fields of industrial control, the requirements of the operation speed and precision are not high, according to the function of the 
electric energy meter verification device and the requirement of the cooperation company, we have chosen the development of QT software based on Linux.

\section{Hardware System Architecture}

\subsection{Selection of master control architecture}

As for the most important part of calibrating device for electric energy meter, we choose the architecture of the core board and expansion board on the hardware. At present, the mainstream processor architectures mainly are X86, DSP, and ARM. In line with high performance, low power consumption, and low cost principle, we decided to adopt the ARM processor AM335X series of architecture. AM335X uses ARM Cortex-A8 architecture, the main frequency of a single kernel up to $800 \mathrm{MHz}$, and it contains LCD, serial port, CAN, USB and other peripherals are also our demand.

\subsection{Hardware system diagram}

The whole electric energy meter calibration device is mainly composed of main controller, standard electric energy meter, signal source, error checking instrument and power amplifier. Fig. 1 is the structure diagram of calibrating device for electric energy meter.

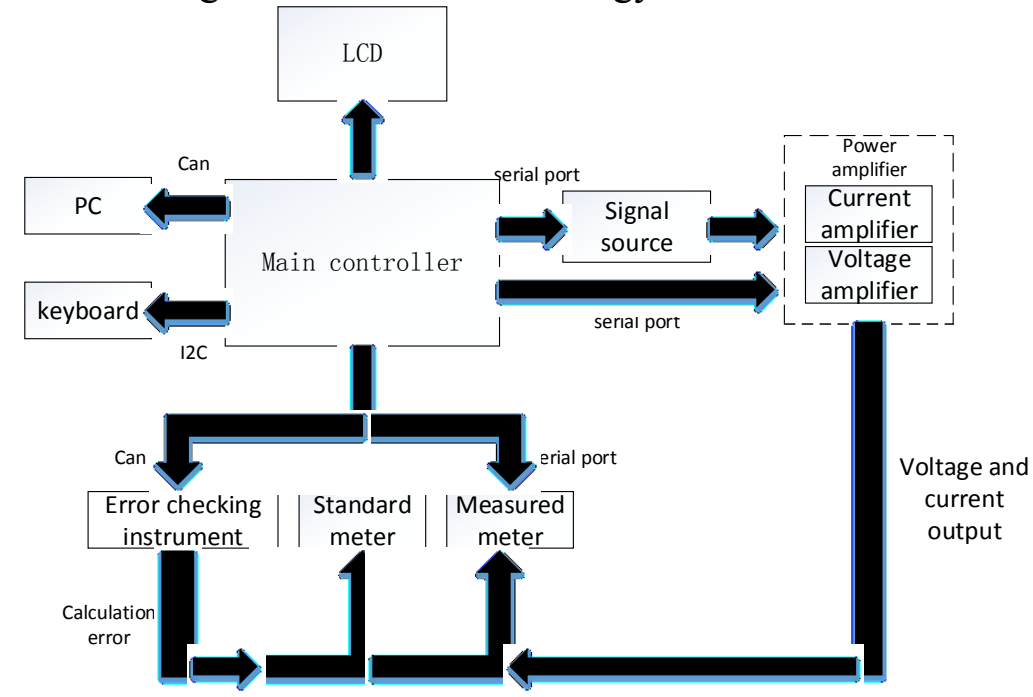

Fig.1 Structure diagram of calibrating device for electric energy meter

The overall work flow of the system are as follows: the main controller is used to control the parts coordinated and orderly work. First of all, we will input the relevant information of the detected meter to the main controller, the voltage and current parameters that need to be generated are transmitted to the signal source, at the same time, the range of voltage amplifier and current amplifier, and the range of standard electric energy meter also need to be switched. After the parameters are obtained, the corresponding AC and DC voltage and current are generated, and is used to provide $\mathrm{AC}$ and $\mathrm{DC}$ voltage and current for electric energy meter, then the power amplifier amplifies the signal to obtain the required AC. The standard electric energy meter is an absolutely accurate meter, we keep the checked meter in parallel with the standard electric energy meter so that they are in the same voltage, current and power factor. Finally, the error checking instrument is used to calculate and return the data, and we judge the checked meter to be accurate according to the result.

\section{Software System Design}

Communication is the key part of the whole software, the main controller of calibrating device for electric energy meter communicates with the standard electric energy meter, communicates with the signal source, and communicates with the power amplifier, the serial communication is adopted; the main controller communicates with PC, and communicates with the error checking Instrument, 
CAN communication is adopted. The main controller needs to read the data from each port at any time. For each port, we design the corresponding receive thread to keep it in the state of reading data. Due to long time running, the data will be read incomplete, dislocation and other problems. In this paper, data is read by multiple reads, each reading only 8 bytes of data and then placed in the cache array until the reception has been met. Fig. 2 is the overall frame of software system.

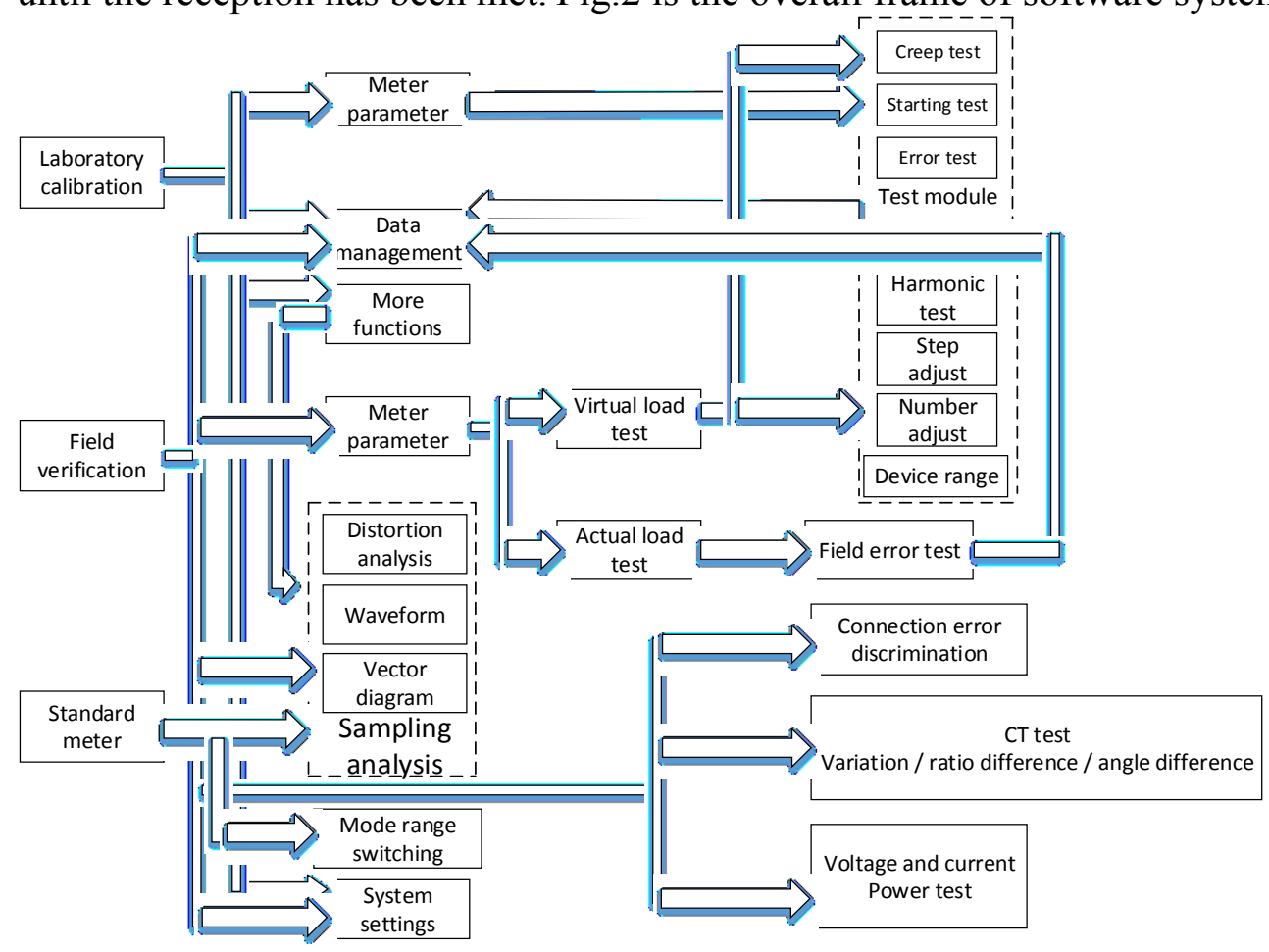

Fig.2 The overall frame of software system

According to the industrial requirements of testing equipment for electric energy meter, verification is mainly divided into: basic error test, creep test, starting test, drop test, and harmonic analysis. Therefore, the software is divided into several sections according to the function. With too many interfaces, we use QT's stack box to put similar interfaces together, the dialog box is divided into the basic error verification, the harmonic analysis, the drop test, the measuring range switching, the number adjust and the step adjust. Fig.3 is patial screenshot of QT's interface, Fig.4 is field test drawing of the main controller for calibrating meter.

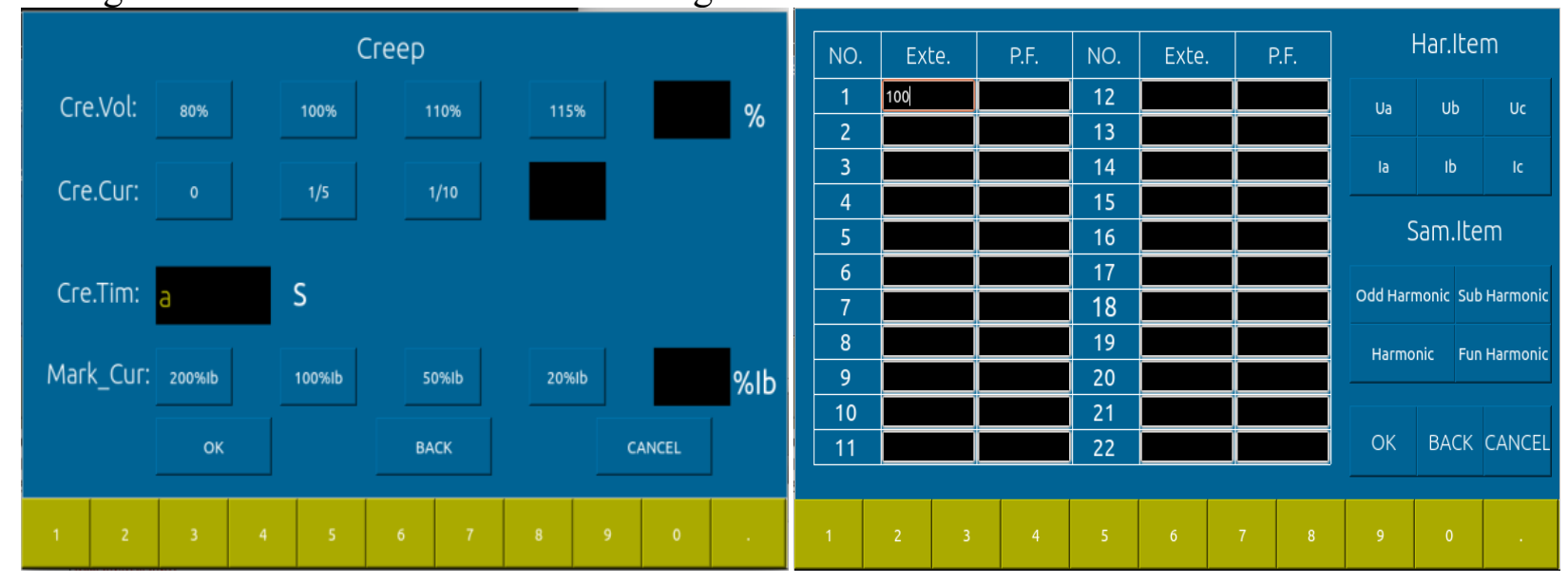

Fig.3 Partial screenshot of QT's interface 


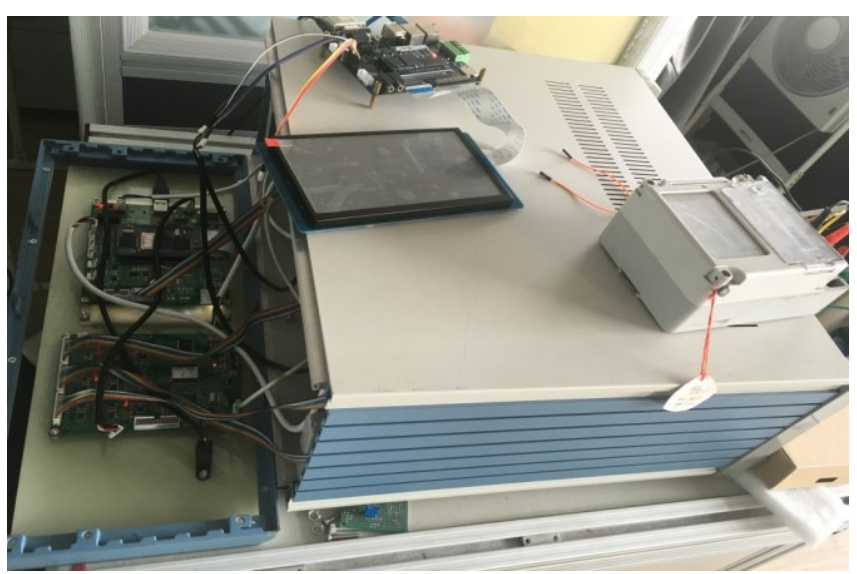

Fig.4 Field test drawing of the main controller for calibrating meter

The basic error of an electric energy meter refers to the relative error value of an electric energy meter under the specified test conditions (including the range of influence quantity, environmental conditions, and test wiring), which reflects the basic accuracy of the energy meter measurement.

The creeping test refers to the circuit of the $0 \%$ rated current and the $80 \%-100 \%$ rated voltage, within the stipulated time meter will produce a pulse, the dial turned a circle. Starting test refers to the circuit of the $80 \%-100 \%$ rated voltage and the $0.1 \% \mathrm{Ib}-0.5 \% \mathrm{Ib}$ rated current, in the specified time, the number of turns of the meter must be greater than a circle.

In addition, the energy meter does not operate at standard sinusoidal waves all the time in the actual grid. At some point the voltage drops abruptly to 0 , and immediately back to its original shape, or to a certain pattern. The state also has rules for the work of electricity meters in these circumstances. In order to simulate the above grid fluctuations, our verification device also has such a drop test environment.

\section{Results}

After one year of research and design, the main control software of calibrating device for the electric energy meter has been preliminarily completed. The calibration items can be carried out normally and the basic error data can be received correctly. For example, we choose the electric energy meter of rated voltage $220 \mathrm{~V}$ and rated current $5 \mathrm{~A}$, and use PC software provided by the manufacturer, the main controller and the PC serial port are connected together to obtain the basic error data of each load point. Table 1 is Partial basic error data of each load point.

Table 1 Partial basic error data of each load point

\begin{tabular}{llll}
\hline & Meter 1 & Meter 2 & Meter 3 \\
\hline All 1.0 Imax & +0.008 & +0.007 & -0.003 \\
All 0.5L Imax & +0.023 & +0.006 & +0.015 \\
All 0.8C Imax & -0.002 & -0.0012 & +0.005 \\
All 1.0 Ib & +0.027 & +0.013 & -0.011 \\
All 0.5C Ib & +0.002 & -0.009 & +0.010 \\
All 0.25L 0.5Ib & -0.012 & -0.026 & +0.014 \\
A 1.0 Imax & -0.016 & -0.010 & -0.019 \\
A 1.0 0.1Ib & -0.012 & -0.014 & +0.006 \\
B 1.0 Ib & +0.017 & -0.001 & +0.004 \\
B 1.0 Imax & +0.007 & -0.015 & -0.018 \\
\hline
\end{tabular}

\section{Conclusions}

The software of the verification device has basically realized the functions required by the cooperative factory, the speed, accuracy and stability of the test are all in line with the requirements. The accuracy of the device is relatively high, reaches the grade of 0.05 . In addition, the interface is more beautiful and easy to operator. Because of the adoption of Linux system, it is also convenient 
for upgrading the later program. In combination with the actual debugging process, we believe that the software design has the following aspects to be improved.

(1) Because of the more control, the jump interface is slow, this aspect needs to be improved.

(2) Taking into account the LCD screen virtual keyboard can't meet the needs of users, later, we can make an external keyboard through the $\mathrm{I} 2 \mathrm{C}$ bus or serial port.

(3) In addition, the accuracy of the device can be higher, the speed of verification can be faster, and the communication mode and the communication speed are all we need to consider.

\section{Acknowledgement}

This work was supported by National Natural Science Foundation of China (U1609216).

\section{References}

[1] Chuanling C, Xian Y, Geng M, et al. Design of the high accuracy reference energy meter $(0.02$ degree)[J]. Electrical Measurement \& Instrumentation, 2003, 11: 006.

[2] Moulin E, d Electricite S, Dictionary S. Measuring reactive power in energy meters[J]. Metering International, 2002, 1(1): 52-54.

[3] Sauter T, Lobashov M. End-to-end communication architecture for smart grids[J]. Industrial Electronics, IEEE Transactions on, 2011, 58(4): 1218-1228.

[4] Wang S, Zhou H, Yuan R-m, et al. Concept and application of smart meter[J]. Power System Technology, 2010, 34: 17-23.

[5] Qing Z, Yan X, Wei H. Digital frequency modulation of programmable signal supply[J]. Electrical Measurement \& Instrumentation, 2001, 8: 008.

[6] Tipsuwan Y, Chow M-Y. Control methodologies in networked control systems[J]. Control engineering practice, 2003, 11(10): 1099-1111.

[7] San Qiang Wang, Xing Zhe Hou, Yan Lin Liu, Qiu Hui Zhuang. Electronic Type Electric Energy Meter Calibrating Method Application Research[J]. Applied Mechanics and Materials, 2013, 2218(278):.

[8] Shuan Ji Zhang, Yu Jie Xia, Min Zhe Zhang. Software Design and Prepayment of Intelligent Electric Meter[J]. Advanced Materials Research, 2013,2526(753):.

[9] Khiewlamyong A, Pirak C. Loop Antenna Design for Smart Energy Meter[C]. Bangkok,Thailand: 2011. 5.

[10] Arghya Sarkar,S.Sengupta.Design and Implemention of a Low Cost Fault Tolerant Three Phase Energy Meter[J].Measurement,2008,41(9). 